\title{
Use of a chemically induced-colon carcinogenesis-prone Apc-mutant rat in a chemotherapeutic bioassay
}

\author{
Kazuto Yoshimi ${ }^{1}$, Takao Hashimoto ${ }^{2}$, Yusuke Niwa² ${ }^{2}$ Kazuya Hata², Tadao Serikawa', Takuji Tanaka ${ }^{3}$
} and Takashi Kuramoto ${ }^{1^{*}}$

\begin{abstract}
Background: Chemotherapeutic bioassay for colorectal cancer (CRC) with a rat model bearing chemically-induced CRCs plays an important role in the development of new anti-tumor drugs and regimens. Although several protocols to induce CRCs have been developed, the incidence and number of CRCs are not much enough for the efficient bioassay. Recently, we established the very efficient system to induce CRCs with a chemically induced-colon carcinogenesis-prone Apc-mutant rat, Kyoto Apc Delta (KAD) rat. Here, we applied the KAD rat to the chemotherapeutic bioassay for CRC and showed the utility of the KAD rat.

Methods: The KAD rat has been developed by the ENU mutagenesis and carries a homozygous nonsense mutation in the Apc gene (S2523X). Male KAD rats were given a single subcutaneous injection of AOM (20 mg/kg body weight) at 5 weeks of age. Starting at 1 week after the AOM injection, they were given 2\% DSS in drinking water for 7 days. Tumor-bearing KAD rats were divided into experimental and control groups on the basis of the number of tumors observed by endoscopy at week 8. The 5-fluorouracil (5-FU) was administrated intravenously a dose of 50 or $75 \mathrm{mg} / \mathrm{kg}$ weekly at week 9, 10, and 11. After one-week interval, the 5-FU was given again at week 13,14 , and 15 . At week 16, animals were sacrificed and tumor number and volume were measured macroscopically and microscopically.
\end{abstract}

Results: In total 48 tumors were observed in 27 KAD rats with a 100\% incidence at week 8 . The maximum tolerated dose for the KAD rat was $50 \mathrm{mg} / \mathrm{kg}$ of 5-FU. Macroscopically, the number or volume of tumors in the 5 -FU treated rats was not significantly different from the control. Microscopically, the number of adenocarcinoma in the 5 -FU treated rats was not significantly different $(p<0.02)$ from that of the control. However, the volume of adenocarcinomas was significantly lower than in the control. Anticancer effect of the 5-FU could be obtained only after the 16 weeks of experimental period.

Conclusion: The use of the AOM/DSS-treated tumor-bearing KAD rats could shorten the experimental period and reduce the number of animals examined in the chemotherapeutic bioassay. The efficient bioassay with the AOM/ DSS-treated tumor-bearing KAD rats would promote the development of new anti-tumor drugs and regimens.

Keywords: Adenomatous polyposis coli, Colorectal cancer, Endoscopy, Rat, Chemotherapy, 5-fluorouracil

\footnotetext{
* Correspondence: tkuramot@anim.med.kyoto-u.ac.jp

${ }^{1}$ Institute of Laboratory Animals, Graduate School of Medicine, Kyoto

University, Yoshidakonoe-cho, Sakyo-ku, Kyoto 606-8501, Japan

Full list of author information is available at the end of the article
} 


\section{Background}

Chemotherapeutic bioassays for colorectal cancer (CRC) play an important role in the development of new antitumor drugs and regimens. These bioassays involve the use of colon carcinogenesis models which mainly consist of animal xenografts, an adenomatous polyposis coli $(A p c)$-mutant mouse model and a chemically-induced CRC model [1-3].

The xenograft model utilizes cultured or primary CRC cells that are implanted under the skin of immunedeficient mice and rats. The size and volume of tumors can be estimated easily and temporally by measuring their dimensions. However, these animals have defects in the immune system that suppresses tumor growth. The subcutaneous microenvironment around the transplanted tumors differs from the colon environment in which the original CRC of the cell lines arose. Therefore, this approach appears to ignore the contribution of the tumor microenvironment and does not exactly mimic tumor development in man $[4,5]$.

Apc-mutant mouse models, such as the Min mouse model, spontaneously develop a considerable number of intestinal tumors and have been widely used as a relevant model for evaluating human chemopreventative therapies. However, tumors in the colon are developed at a much lower frequency than in the small intestine. Even if tumors do develop in the colon, almost all of them are low grade adenomas [6].

The chemically-induced CRC model is superior to these models in that the characteristics of the induced tumor are very similar to those of human CRC. Tumors only develop in the colon through multi-step carcinogenesis which mimics the entire process of tumor growth in man. In this model, tumor morphology and mutation spectrum are also similar to those in human CRC [6]. Moreover, methods of inducing colon tumors are well-established, so that we can be certain of obtaining the number of tumors expected, which is ideal for the evaluation of potential chemotherapeutic drugs $[2,7]$.

Although many carcinogens induce colon tumors in rats, azoxymethane (AOM) administered subcutaneously has been most widely used $[2,6,8]$. However, the incidence of colon tumors induced by two or three subcutaneous injections of AOM is not high, and it takes 7-9 months to induce sufficient tumors to evaluate the chemotherapeutic efficacy of potential anti-cancer drugs [9]. Such limitations have been significantly improved by using dextran sodium sulfate (DSS) as an inflammatory agent. When 2\% DSS is administered in drinking water to the AOM-treated rats for one week, starting one week after administration, a number of colon tumors develop within a short time period (this is known as the TANAKA method) [10].
Recently we developed a novel Apc mutant rat strain, called the Kyoto Apc Delta (KAD) rat (strain name: F344- $A p c^{m 1 k y o}$ ) from our ENU-mutagenesis program. The KAD rat carries a homozygous nonsense mutation in the Apc gene (S2523X). Thus, the KAD rat lacks 321amino acids in the C-terminal of APC, but it remains viable at almost 2 years and shows no spontaneous colorectal tumors. Moreover, by applying the TANAKA method to KAD rats, we obtained a much higher incidence, multiplicity and malignancy of colon tumors in KAD rats than colon tumors in F344 wild rats. We were able to induce these tumors within 15 weeks of the experimental period. In addition, we were able to carry out endoscopic observation, by which colon tumors could be detected from Week 8 [11].

In the present study, in order to establish an efficient chemotherapeutic bioassay with KAD rats, we induced colon tumors by means of treatment with AOM and DSS, and then administered a typical anti-tumor drug, namely 5 -fluorouracil (5-FU) to the tumor-bearing rats.

\section{Methods \\ Chemicals}

5-FU was purchased from Kyowa Hakko Kogyo, Co., Ltd. (Tokyo, Japan). AOM was purchased from SigmaAldrich Chemical Co. (St. Louis, MO, USA). These drugs were diluted in saline just before administration. DSS (MW 36,000-50,000) was purchased from ICN Biochemicals, Inc. (Aurora, OH, USA). DSS was dissolved in distilled water at $2 \%(\mathrm{w} / \mathrm{v})$ every day before treatment.

\section{Rats}

Specific pathogen free male KAD rats were purchased from Japan SLC, Inc. (Hamamatsu, Japan) and provided by the National Bio Resource Project for the Rat (http:// www.anim.med.kyoto-u.ac.jp/nbr) at 4 weeks of age. The rats were acclimatized for a week before the experiment and were maintained under conditions of $50 \pm 10 \%$ humidity, $12 \mathrm{~h}-12 \mathrm{~h}$ light cycle and $24 \pm 2{ }^{\circ} \mathrm{C}$ temperature. They were fed a standard pellet diet (F-2, Funabashi Farm, Funabashi, Japan) and tap water ad libitum.

\section{Induction of colon tumor}

Chemically induced-colon carcinogenesis was carried out as described in our previous study [11]. Briefly, male KAD rats $(\mathrm{n}=32)$ were given a single subcutaneous injection of AOM (20 mg/kg body weight) at 5 weeks of age. Starting at 1 week after the AOM injection, they were given $2 \%$ DSS in drinking water for 7 days (Figure 1). Five rats were used to find correlation of the number of polypoid lesions with the volume of tumors at Week 8. All experimental procedures were approved by the Animal Research Committee of Kyoto University 


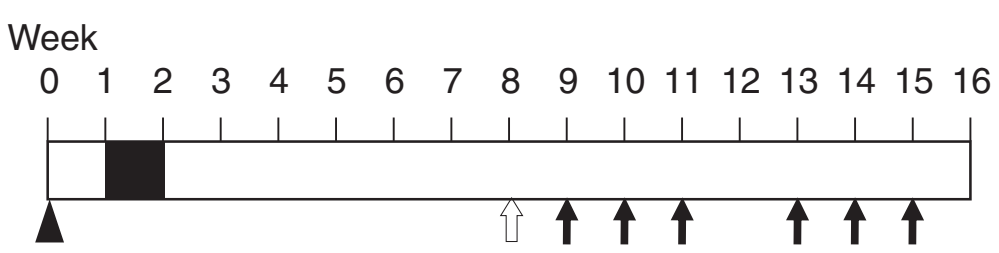

Figure 1 Experimental schedule. KAD rats at 5 weeks of age were given a subcutaneous injection of AOM at $20 \mathrm{mg} / \mathrm{kg}$ body weight (arrow head). One week after the AOM injection they were given 2\% DSS (MW 36,000-50,000) in their drinking water for one week (black box). Endoscopic observation was carried out at week 8 (open arrow). 5-FU was administrated intravenously at weeks 9, 10, 11, 13, 14 and 15 (arrows). All animals were sacrificed at week 16.

and were performed according to the Regulation on Animal Experimentation at Kyoto University.

\section{Endoscopic observation}

Observation was performed at week 8 with an endoscope (BF TYPE 3C40: Olympus, Tokyo, Japan) to determine the presence of colon tumors (Figure 1). KAD rats were anesthetized by administration of $2 \%$ isoflurane (Forane: Abbott Japan, Tokyo, Japan) vapor through a nose cone. The colon was flushed using an enema of tap water to remove feces. The endoscope was inserted into the colon, and endoscopic images were acquired within the distal colon and rectum. The numbers of polypoid lesions, assumed to be developing colorectal tumors, were counted.

\section{Chemotherapeutic test}

The AOM/DSS-treated rats were divided into three groups (nine rats each), among which numbers of colon tumors were not significantly different. The 5-FU was administrated to the tumor-bearing KAD rats at two different doses (50 or $75 \mathrm{mg} / \mathrm{kg}$ ) by three weekly intravenous (i.v.) injections at weeks 9, 10 and 11. According to the preliminary experiment, we set a 1 week withdrawal period to decrease the occurrence of serious side effects caused by 5 -FU. One week later, rats underwent additional administration of 5-FU involving three weekly i.v. injections at weeks 13,14 and 15. At week 16 animals were sacrificed by cervical dislocation under anesthesia with isoflurane (Figure 1). Then the colorectum of the rats was resected, washed with PBS, opened longitudinally along the main axis and fixed in $10 \%$ neutral buffered formalin for at least $24 \mathrm{~h}$. The number and volume of colon tumors were measured after fixation. The other organs such as small intestine, stomach, liver and kidney were observed macroscopically for any abnormalities.

\section{Histopathological examination}

After careful macroscopic inspection, tumors and whole colonic mucosa were embedded in paraffin and sectioned for histopathology after staining with hematoxylin and eosin. After tumors that developed in the colorectum were photographed, the largest and the smallest superficial diameters of adenocarcinoma that were diagnosed histopathologically were measured on the photographs. Tumor volume was calculated according to the formula $\mathrm{V}=\mathrm{a} \times \mathrm{b}^{2} / 2$, in which "a" is the largest superficial diameter and " $b$ " is the smallest superficial diameter [12].

\section{Immunohistochemistry}

Cell proliferation and apoptosis were evaluated by determination of the percentages of PCNA- and cleaved caspase-3-positive nuclei in a total of 200 cancer cells for each sample ( $n=6$ from the control group and $n=8$ from Group 1). Briefly, sections were incubated with antimouse PCNA antibody (clone PC10, 1:1000 dilution; DAKO) and cleaved caspase-3 (Asp175) antibody (1:1000 dilution; Cell Signaling Technology) overnight at $4{ }^{\circ} \mathrm{C}$. Biotinyl antibody was used as secondary antibody and then the streptavidin-peroxidase complex $\left(\mathrm{LASB}^{\mathrm{TM}}+\mathrm{Kit}\right.$, Universal, DAKO) was applied. The antigen-antibody complex was visualized by 3,3/-diamonobenzidine tetrachloride (DAKO).

\section{Statistical analysis}

Data are expressed as the mean \pm standard deviation (S.D.). Student's $t$-test was performed using the statistics package within Microsoft Excel for statistical analysis, and $\mathrm{p}$ values were considered significant when $<0.05$.

\section{Results}

Correlation of the number of polypoid lesions with the total volume of tumors

To find the correlation of the number of polypoid lesions with the total volume of tumors, we induced colon tumors to KAD rats $(n=5)$ by the TANAKA method and counted tumor number under the endoscopy and the number and volume of tumors under the microscopy at week 8 (Additional file 1: Table S1). As a good correlation between them was found, it is very likely that the number of polypoid lesions found with the endoscopy at week 8 can be used to estimate the total volume of tumors (Additional file 2: Figure S1). 


\section{Effective tumor development in AOM/DSS-treated KAD rats}

At week 8 when carrying out endoscopic observations for the occurrence of colon tumors in the colons of AOM/DSS-treated KAD rats, we could observe about $10 \mathrm{~cm}$ of the luminal surface, from the rectum to the distal colon. We found polypoid lesions around the rectum and the distal colon. Polypoid lesions which were clearly different from normal mucosa assumed to be developing colorectal tumors. All AOM/DSS-treated $\mathrm{KAD}$ rats developed colon tumors. In total 48 tumors were observed in $27 \mathrm{KAD}$ rats with a $100 \%$ incidence and a multiplicity of $1.78 \pm 0.85$, ranging from 1 to 4 per rat.

\section{Dosing condition of 5-FU}

On the basis of the number of tumors, the tumorbearing KAD rats were divided into three groups. One was the control group and the others were experimental groups, in which rats were given 5 -FU at a concentration of $50 \mathrm{mg} / \mathrm{kg}$ (Group 1) or $75 \mathrm{mg} / \mathrm{kg}$ (Group 2). Each group consisted of nine rats and the total number of tumors in each group was 16. The average number of tumors per rat was not significantly different among the groups (Control: $1.78 \pm 0.83$, Group 1: $1.78 \pm 0.83$, Group 2: $1.78 \pm 0.97$ ) (Figure 2B).

The average body weight of rats in Group 1 tended to be lower than in the control group, and was significantly different from the control group at weeks 15 (300.0 \pm 18.1 vs $319.4 \pm 20.1 ; \quad \mathrm{p}<0.05)$ and $16 \quad(296.1 \pm 18.9 \quad$ vs $318.8 \pm 18.8 ; \mathrm{p}<0.03)$. However the reduction in body weight was less than $10 \%$ as compared with the control. None of the rats in Group 1 died during the experiment. On the other hand, gain in body weight in Group 2 was constantly and significantly impaired throughout the experimental period. More than $10 \%$ of weight loss was observed at weeks 12, 15 and 16, as compared with that in the control group (Figure 3A). KAD rats in Group 2 had severe bloody stools and diarrhea and six rats (67\%) in the group died during the experiment (Figure 3B). These findings indicated that the $75 \mathrm{mg} / \mathrm{kg}$ dose of 5 -FU was too toxic for the tumor-bearing KAD rats, and led to the marked body weight loss and eventually to death. Thus, the $50 \mathrm{mg} / \mathrm{kg}$ dose of 5 -FU was considered to be appropriate for evaluation of the antitumor activity of 5-FU, when we used the tumor-bearing KAD rats in a chemotherapeutic bioassay.

\section{Reduction of volume but not number of adenocarcinomas in the tumor-bearing KAD rats by treatment with 5-FU}

At week 16 we carried out an autopsy and macroscopic examination of the large bowels of the control group and Group 1. Macroscopically, rats in both groups developed multiple nodular, polypoid or caterpillar-like tumors mainly in the rectum and distal colon (Figure 4). The number of tumors in Group 1 was not significantly different from the control group (Table 1). The volume of tumors, which were macroscopically calculated, was $27 \%$ smaller in Group 1 than in the control group, but the difference was not significant $(\mathrm{p}=0.34)$.

Microscopically, all tumors that developed in KAD rats were tubular adenoma or well- or moderatelydifferentiated tubular adenocarcinoma (Figure 5A and $5 \mathrm{~B})$. The multiplicity of adenoma or adenocarcinoma in Group 1 was not significantly different from that of the control group $(\mathrm{p}=0.53 ; \mathrm{p}=0.44$, respectively) (Table 1$)$. The size of the adenomas was too small for their volumes to be calculated. However, the volume of adenocarcinomas $\left(63.85 \pm 51.06 \mathrm{~mm}^{3}\right)$ in Group 1 was significantly lower $(\mathrm{p}<0.02)$ than in the control group $\left(34.40 \pm 31.26 \mathrm{~mm}^{3}\right)$, when the volume was calculated from the histological sections. In addition, we found significant reduction of PCNA labeling index as well as
A

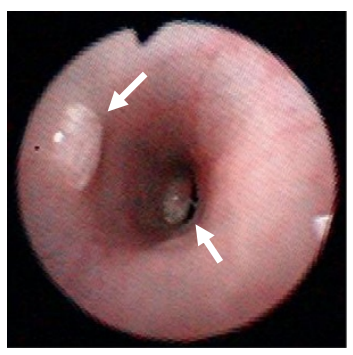

B

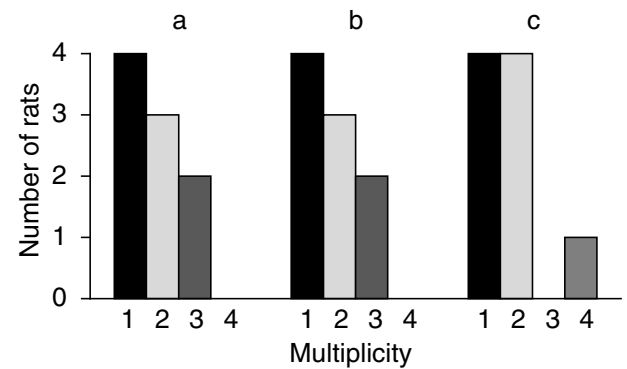

Figure 2 Grouping of AOM/DSS-treated KAD rats before 5-FU treatment. (A) Endoscopic view of colon tumors (arrows) in an AOM/DSStreated KAD rat at week 8. (B) AOM/DSS-treated KAD rats were divided into experimental groups based on the number of tumors induced in their colons. The number of tumors induced in each animal determined by endoscopic observations varied from one to four. In total, 48 tumors were found in 27 rats. The tumor-bearing rats (nine per group) were divided into three groups (a: saline, b: 50 mg/kg 5-FU and c: 75 mg/ $\mathrm{kg}$ 5-FU), so as not to be significantly different at the starting point of treatments. 

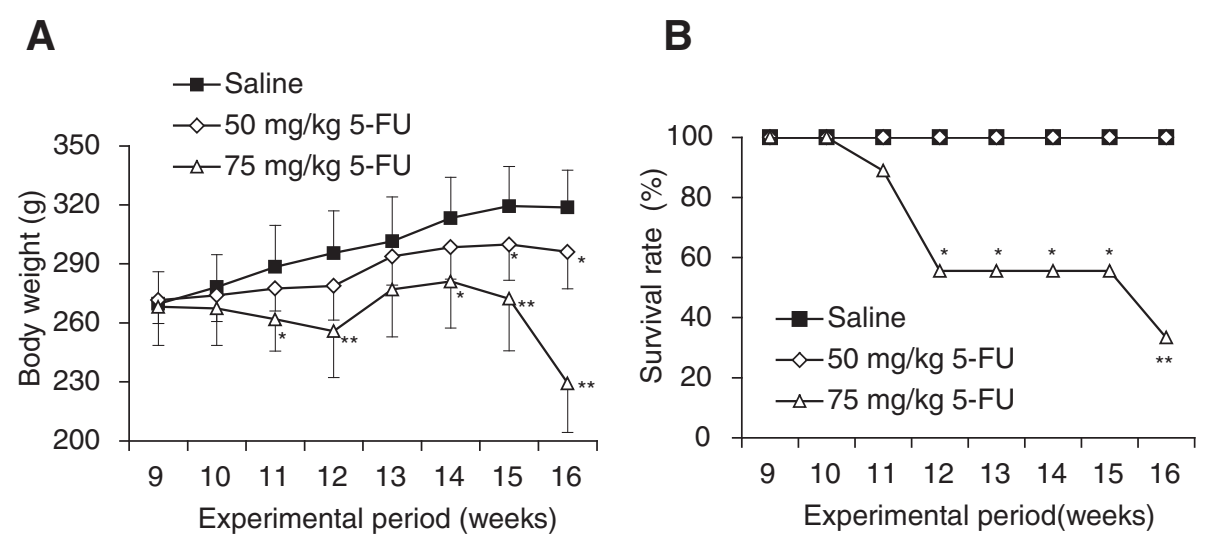

Figure 3 Toxicity of 5-FU in AOM/DSS treated KAD rats. Average body weight (A) and survival rate (B) of the control, $50 \mathrm{mg} / \mathrm{kg}$ and $75 \mathrm{mg} /$ kg 5-FU treated groups. ${ }^{*} p<0.05,{ }^{* *} p<0.001$.

significant elevation of cleaved caspase-3 positive rate in the adenocarcinomas in Group 1 (Figure 6). These results suggest that the 5-FU treatment suppressed cell proliferation and induced apoptosis and thereby inhibited adenocarcinoma development.

\section{Discussion}

Carcinogenic process is complex. Tumor development proceeds via a multi-step process, in which a succession of genetic changes, each conferring one or another type of growth advantage, leads to the progressive conversion of normal cells into cancer cells. Moreover, extent of cell transformation depends on the genetic predisposition and environmental factors [13]. Thus, to obtain cancerous lesions effectively, it is necessary to use a synergy effect of genetic and environmental factors. Our carcinogenic system with KAD rats employs such synergy effect of Apc-mutation, chemical carcinogen exposure, and tissue inflammation.

In ideal chemotherapeutic bioassay systems, the number and volume of tumors should be evaluated as the indicator of anti-tumor drug efficacy. Therefore, it is indispensable to be able to strictly set the size of the experimental and control groups, among which the number and volume of tumors should not differ significantly. To this end, we carried out endoscopic observations in the colons of AOM/DSS-treated KAD rats, and divided animals into groups on the basis of the number of colon tumors. Since the rat has a suitable body size for handling, we could easily manipulate the endoscope and correctly count the number of tumors. At week 8 we found colon tumors with a $100 \%$ incidence in AOM/DSStreated KAD rats. The rats developed one to four tumors. On the basis of the number of tumors, we could set the experimental and control groups, because
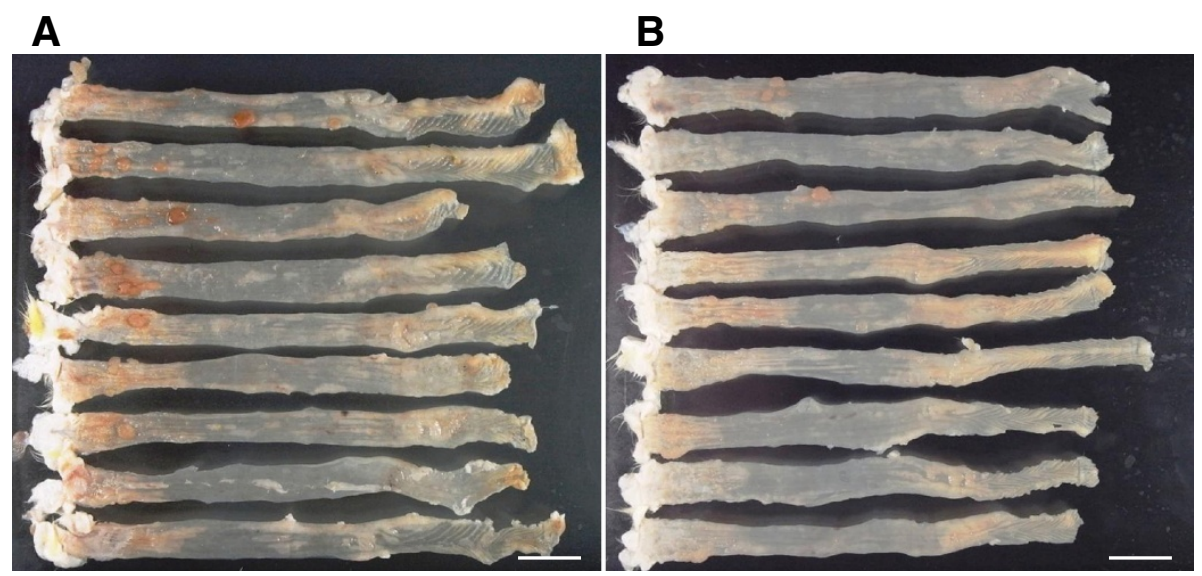

Figure 4 Macroscopic view of large bowel. Macroscopic view of large bowel of KAD rats that were not given 5-FU (A) and that were given $50 \mathrm{mg} / \mathrm{kg} 5-\mathrm{FU}$ (B). Colon tumors that developed in both groups were mainly distributed in the rectum and distal colon, which was assumed to be within $8 \mathrm{~cm}$ from the anus. No tumors were observed in the proximal colon. Left side: anus. Right side: cecum. Bar: $2 \mathrm{~cm}$. 
Table 1 Effects of 5-FU on the development of colon tumors in the KAD rat

\begin{tabular}{|c|c|c|c|c|c|c|c|}
\hline \multirow[t]{3}{*}{ Treatment } & \multirow[t]{3}{*}{ No. of rats } & \multicolumn{2}{|c|}{ Macroscopic observation } & \multicolumn{4}{|c|}{ Microscopic observation } \\
\hline & & \multirow[t]{2}{*}{ Multiplicity } & \multirow[t]{2}{*}{ Volume $\left(\mathrm{mm}^{3}\right)^{1}$} & \multicolumn{3}{|c|}{ Multiplicity } & \multirow{2}{*}{$\frac{\text { Volume }\left(\mathrm{mm}^{3}\right)^{2}}{\text { adenocarcinoma }}$} \\
\hline & & & & adenomas & adenocarcinomas & total & \\
\hline Saline & 9 & $5.56 \pm 3.43$ & $106.34 \pm 68.92$ & $3.11 \pm 2.52$ & $3.22 \pm 2.77$ & $6.33 \pm 4.87$ & $63.85 \pm 51.06$ \\
\hline 50 mg/kg 5-FU & 9 & $6.33 \pm 3.04$ & $77.28 \pm 57.23$ & $3.78 \pm 1.79$ & $2.33 \pm 1.94$ & $6.11 \pm 2.37$ & $34.40 \pm 31.26^{3}$ \\
\hline
\end{tabular}

${ }^{1}$ Tumor volume was determined by the formula $V=a \times b^{2} / 2$ ( $V$ : volume. a: the largest superficial diameter and $b$ : the smallest superficial diameter).

${ }^{2}$ Volumes of adenomas were too small to calculate.

${ }^{3}$ Adenocarcinoma volumes observed in the 5 -FU-treated KAD rats were significantly reduced as compared with those of non-treated rats ( $p<0.02$ ).

the number of tumors observed by the endoscopy is correlated to the volume of tumors obtained by the microscopy at week 8 . We, therefore, recommend counting the number of tumors using endoscopic observation before dividing the rats into groups.

It is important to identify biomarkers that are used to predict efficacy and safety of anti-tumor drugs. Rats can be subjected to the sequential sampling of bloods. The amounts of bloods or urines are enough to be examined. Moreover, drug kinetics can be monitored by in vivo imaging [14]. Thus, the chemotherapeutic bioassay with the KAD rats is a candidate system to explore the biomarkers.

5-FU is a pyrimidine analog and when incorporated into DNA inhibits the cell's ability to synthesize DNA. Eventually 5-FU induces cell cycle arrest and apoptosis, mainly in cells with high proliferative activity such as cancer cells [15]. Side effects of 5-FU, such as diarrhea and weight loss, are problematic in performing chemotherapeutic tests with animal models. Thus, it is important to determine the maximum tolerated dose (MTD) that does not produce profound weight loss, and that causes no drug-related lethality. Usually the MTD of 5 -FU in rats ranges from 25 to $100 \mathrm{mg} / \mathrm{kg}$, depending on the 5-FU administration schedules [16]. In the current study, we found that the MTD was $50 \mathrm{mg} / \mathrm{kg}$ of 5-FU when administered to tumor-bearing KAD rats by i.v. injection. Although the MTD should be determined using different administration schedules and routes, the MTD that we determined in the present study can be a helpful guide in setting doses of anti-cancer drugs in further chemotherapeutic tests with KAD rats.

In our study, the treatment of tumor-bearing KAD rats with 5-FU failed to reduce the multiplicity of adenoma or adenocarcinoma. However, the treatment significantly reduced adenocarcinoma tumor volume and cell proliferation as well as increased adenocarcinoma apoptosis, which was consistent with the mode of action of the 5-FU [15]. Treatment response assessed in terms of

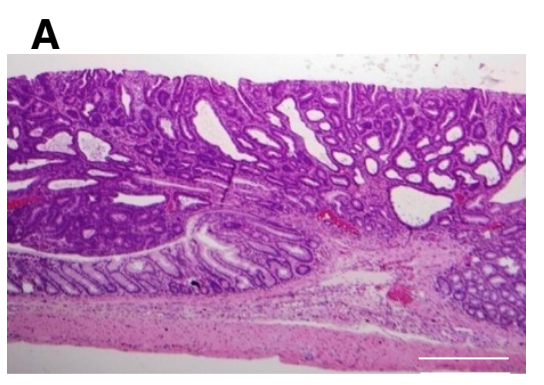

B

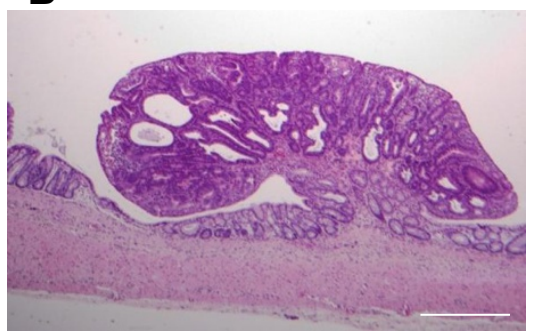

\section{C}

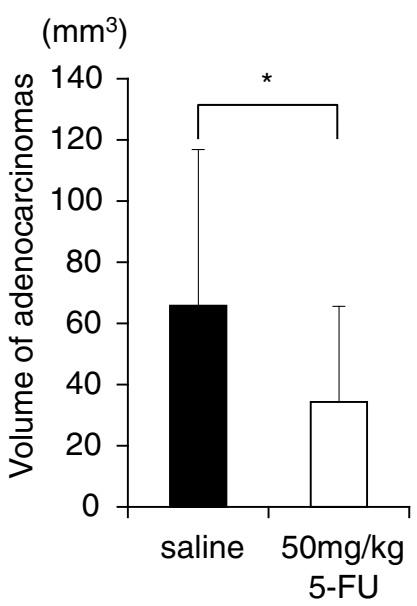

Figure $\mathbf{5}$ Histopathological analysis of colonic adenocarcinomas. Colonic adenocarcinoma developed in KAD rats that were not given 5-FU (A) and that were given $50 \mathrm{mg} / \mathrm{kg} 5$-FU (B). Most of the adenocarcinomas in KAD rats that received $50 \mathrm{mg} / \mathrm{kg} 5$-FU were smaller than those in the control group (hematoxylin and eosin stain). Bar: $500 \mu \mathrm{m}$. (C) Average volume of adenocarcinoma tumors grown in KAD rats that were given saline or 5 -FU $(50 \mathrm{mg} / \mathrm{kg})$. The mean volume of adenocarcinoma tumors in the 5 -FU-treated rats were significantly smaller than those in the non-treated rats. *: $p<0.02$. 


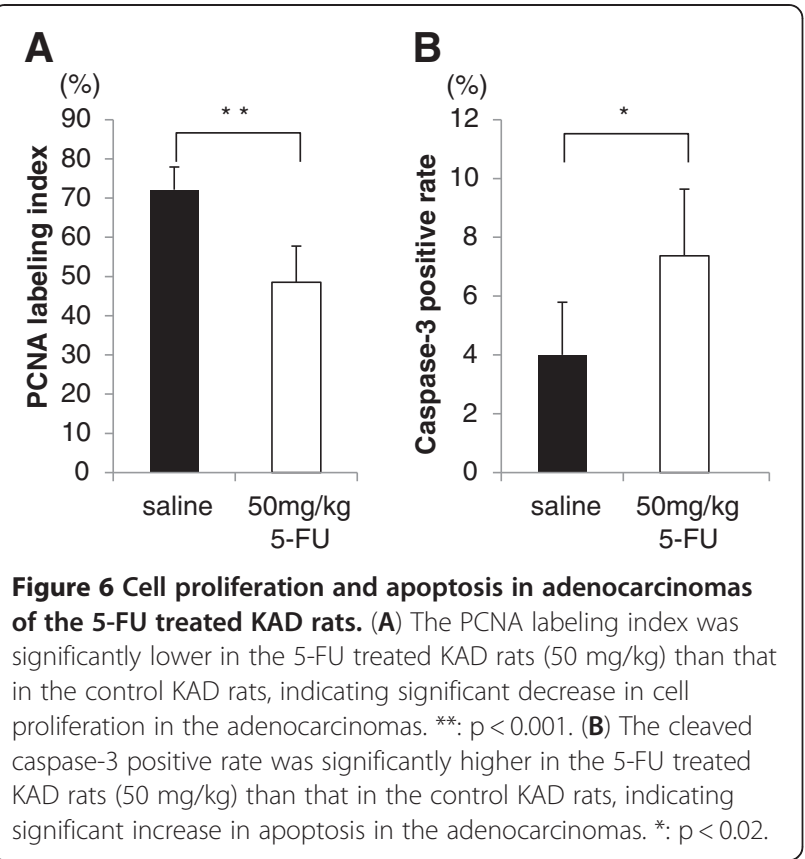

change in tumor size after 5-FU administration in the present study amounted to a $30 \%$ reduction, which was similar to the response rate of 5 - $\mathrm{FU}$ as a single agent seen in human cancers, including CRC [17]. These findings indicated that the response of tumors in AOM/DSS-treated KAD rats to 5-FU treatment was similar to human CRC, and supported the view that this should be a useful bioassay system for employment in further chemotherapeutic studies.

\section{Conclusions}

In the present study we established a chemotherapeutic bioassay system for CRC using KAD rats. In this system, we could set the experimental groups on the basis of the number of tumors detected by endoscopic examination. After 5-FU administration rat colon tumors induced by AOM/DSS treatments showed a similar response, in terms of percentage reduction in size and cell proliferation and percentage elevation in apoptosis, to those reported in clinical CRC studies. Thus, we expect that this system could effectively promote the development of new anti-tumor drugs and regimens for human CRC.

\section{Additional files}

Additional file 1: Table S1. Number and total volume of tumors found in KAD rats at week 8.

Additional file 2: Figure S1. The correlation of total volume of tumors with the numbers of polypoid lesions observed by endoscopy at Week8. Regression formula was made with Excel software package (Microsoft). Vertical axis was shown in logarithmic scale.
Competing interests

The authors declare that they have no competing interests.

\section{Authors' contributions}

KY and TK conceived the study and designed the experiments. KY, TH, YN and $\mathrm{KH}$ performed the experiments. TT performed the histopathological analysis. KY, TK and TT wrote the manuscript. TS revised the manuscript. All authors have read and approved the final manuscript.

\section{Acknowledgments}

This work was supported in part by a Grant-in-Aid for Cancer Research from the Ministry of Health, Labour and Welfare (to TK), and Grants-in-Aid for Scientific Research from the Japan Society for the Promotion of Science (21300153 to TK and 0233639 to KY). The KAD (F344-Apc ${ }^{\text {miKyo }}$ ) rat has been deposited in the National BioResource Project-Rat in Japan and is available from the Project (http://www.anim.med.kyoto-u.ac.jp/nbr)

\section{Author details}

${ }^{1}$ Institute of Laboratory Animals, Graduate School of Medicine, Kyoto University, Yoshidakonoe-cho, Sakyo-ku, Kyoto 606-8501, Japan. ${ }^{2}$ Sunplanet Co., Ltd, 4388 Makita, Kamiishizu, Ogaki 503-1602, Japan. ${ }^{3}$ Cancer Research and Prevention, The Tohkai Cytopathology Institute, 4-33 Minami-Uzura, Gifu 500-8285, Japan

Received: 24 April 2012 Accepted: 26 September 2012

Published: 3 October 2012

\section{References}

1. Blumenthal RD, Osorio L, Hayes MK, Horak ID, Hansen HJ, Goldenberg DM: Carcinoembryonic antigen antibody inhibits lung metastasis and augments chemotherapy in a human colonic carcinoma xenograft. Cancer Immunol Immunother 2005, 54(4):315-327.

2. Corpet DE, Pierre F: Point: From animal models to prevention of colon cancer. Systematic review of chemoprevention in min mice and choice of the model system. Cancer Epidemiol Biomarkers Prev 2003, 12(5):391-400.

3. Williams KJ, Telfer BA, Stratford IJ, Wedge SR: ZD1839 ('Iressa'), a specific oral epidermal growth factor receptor-tyrosine kinase inhibitor, potentiates radiotherapy in a human colorectal cancer xenograft model. Br J Cancer 2002, 86(7):1157-1161.

4. Ding Y, Cravero JD, Adrian K, Grippo P: Modeling pancreatic cancer in vivo: from xenograft and carcinogen-induced systems to genetically engineered mice. Pancreas 2010, 39(3):283-292.

5. Voskoglou-Nomikos T, Pater J, Seymour L: Clinical predictive value of the in vitro cell line, human xenograft, and mouse allograft preclinical cancer models. Clin Cancer Res 2003, 9(11):4227-4239.

6. Femia AP, Caderni G: Rodent models of colon carcinogenesis for the study of chemopreventive activity of natural products. Planta Med 2008, 74(13):1602-1607.

7. Bruce WR: Counterpoint: From animal models to prevention of colon cancer. Criteria for proceeding from preclinical studies and choice of models for prevention studies. Cancer Epidemiol Biomarkers Prev 2003, 12(5):401-404.

8. Reddy BS: Studies with the azoxymethane-rat preclinical model for assessing colon tumor development and chemoprevention. Environ $\mathrm{Mol}$ Mutagen 2004, 44(1):26-35.

9. Reddy BS, Maeura Y: Tumor promotion by dietary fat in azoxymethaneinduced colon carcinogenesis in female F344 rats: influence of amount and source of dietary fat. J Natl Cancer Inst 1984, 72(3):745-750.

10. Tanaka T, Kohno H, Suzuki R, Yamada Y, Sugie S, Mori H: A novel inflammation-related mouse colon carcinogenesis model induced by azoxymethane and dextran sodium sulfate. Cancer Sci 2003, 94(11):965-973.

11. Yoshimi K, Tanaka T, Takizawa A, Kato M, Hirabayashi M, Mashimo T, Serikawa T, Kuramoto T: Enhanced colitis-associated colon carcinogenesis in a novel Apc mutant rat. Cancer Sci 2009, 100(11):2022-2027.

12. Carlsson G, Gullberg B, Hafstrom L: Estimation of liver tumor volume using different formulas - an experimental study in rats. J Cancer Res Clin Oncol 1983, 105(1):20-23.

13. Hanahan D, Weinberg RA: The hallmarks of cancer. Cell 2000, 100(1):57-70.

14. Ogawa K, Mukai T, Kawai K, Takamura N, Hanaoka H, Hashimoto K, Shiba K, Mori $\mathrm{H}$, Saji $\mathrm{H}$ : Usefulness of competitive inhibitors of protein binding for 
improving the pharmacokinetics of 186Re-MAG3-conjugated bisphosphonate (186Re-MAG3-HBP), an agent for treatment of painful bone metastases. Eur J Nucl Med Mol Imaging 2009, 36(1):115-121.

15. Thomas DM, Zalcberg JR: 5 -fluorouracil: a pharmacological paradigm in the use of cytotoxics. Clin Exp Pharmacol Physiol 1998, 25(11):887-895.

16. Cao S, Rustum YM: Synergistic antitumor activity of irinotecan in combination with 5 -fluorouracil in rats bearing advanced colorectal cancer: role of drug sequence and dose. Cancer Res 2000, 60(14):3717-3721.

17. Salonga D, Danenberg KD, Johnson M, Metzger R, Groshen S, Tsao-Wei DD, Lenz HJ, Leichman CG, Leichman L, Diasio RB, et al: Colorectal tumors responding to 5 -fluorouracil have low gene expression levels of dihydropyrimidine dehydrogenase, thymidylate synthase, and thymidine phosphorylase. Clin Cancer Res 2000, 6(4):1322-1327.

doi:10.1186/1471-2407-12-448

Cite this article as: Yoshimi et al:: Use of a chemically induced-colon carcinogenesis-prone Apc-mutant rat in a chemotherapeutic bioassay. BMC Cancer 2012 12:448.

\section{Submit your next manuscript to BioMed Central and take full advantage of:}

- Convenient online submission

- Thorough peer review

- No space constraints or color figure charges

- Immediate publication on acceptance

- Inclusion in PubMed, CAS, Scopus and Google Scholar

- Research which is freely available for redistribution 